\title{
Closed-Form Parameterization of the Pareto Boundary for the Two-User MISO Interference Channel
}

\author{
Johannes Lindblom, Eleftherios Karipidis and Erik G. Larsson
}

\section{Linköping University Post Print}

N.B.: When citing this work, cite the original article.

C2011 IEEE. Personal use of this material is permitted. However, permission to reprint/republish this material for advertising or promotional purposes or for creating new collective works for resale or redistribution to servers or lists, or to reuse any copyrighted component of this work in other works must be obtained from the IEEE.

Johannes Lindblom, Eleftherios Karipidis and Erik G. Larsson, Closed-Form Parameterization of the Pareto Boundary for the Two-User MISO Interference Channel, 2011, Proceedings of the IEEE International Conference on Acoustics, Speech and Signal Processing (ICASSP), 3372-3375. 


\title{
CLOSED-FORM PARAMETERIZATION OF THE PARETO BOUNDARY FOR THE TWO-USER MISO INTERFERENCE CHANNEL
}

\author{
Johannes Lindblom, Eleftherios Karipidis, and Erik G. Larsson \\ Communication Systems Division, Department of Electrical Engineering (ISY) \\ Linköping University, SE-581 83 Linköping, Sweden. \\ \{lindblom, karipidis, erik.larsson\}eisy.liu.se
}

\begin{abstract}
In this paper, we study an achievable rate region of the two-user multiple-input single-output (MISO) interference channel. We find the transmit beamforming vectors that achieve Pareto-optimal points. We do so, by deriving a sufficient condition for Pareto optimality. Given the beamforming vector of one transmitter, this condition enables us to determine the beamforming vector of the other transmitter that forms a Pareto-optimal pair. The latter can be done in closed form by solving a cubic equation. The result is validated against state-of-the-art methods via numerical illustrations.
\end{abstract}

Index Terms - Beamforming, interference channel, multipleinput single-output (MISO), Pareto optimality, rate region

\section{INTRODUCTION}

We consider the scenario where two wireless transmitter (TX) - receiver $(\mathrm{RX})$ pairs operate simultaneously at the same frequency. The two pairs, i.e., $\mathrm{TX}_{1} \rightarrow \mathrm{RX}_{1}$ and $\mathrm{TX}_{2} \rightarrow \mathrm{RX}_{2}$, are located in the proximity of each other. Therefore, they mutually interfere each other. This setup is known as the interference channel (IC). In this paper, we assume that the TXs are equipped with $n \geq 2$ antennas, whereas the RXs are equipped with a single antenna each. Hence, this is a multiple-input single-output (MISO) IC [1]. We assume that the RXs treat interference as noise and that the TXs have perfect channel state information. The focus of this paper is to propose an efficient method for finding the Pareto, i.e., outer, boundary of the rate region. We build upon the fact that this is a one-to-one curve in the two-dimensional space, so it can be described using one real-valued parameter. We derive a relation that couples the transmit strategies, here beamforming vectors, in such way that they together achieve a Pareto-optimal (PO) operating point.

A single real-scalar parameter to characterize the family of beamforming vectors that can potentially be PO was introduced in [1]. This parameterization only provides necessary conditions that each TX has to separately fulfill to achieve a PO point. A bruteforce pairing of the TX strategies, that the parameterization yields, is needed to find the boundary. Nevertheless, the results of [1] provided tools for analyzing the MISO IC. In [2] the concept of virtual signal-to-interference-plus-noise ratio (SINR) was used to obtain

This work has been supported in part by the Swedish Research Council (VR), the Swedish Foundation of Strategic Research (SSF), and the Excellence Center at Linköping-Lund in Information Technology (ELLIIT). This work has been performed in the framework of the European research project SAPHYRE, which is partly funded by the European Union under its FP7 ICT Objective 1.1 - The Network of the Future. E. G. Larsson is a Royal Swedish Academy of Sciences (KVA) Research Fellow supported by a grant from Knut and Alice Wallenberg Foundation. an alternative characterization of the Pareto boundary. General interference networks were studied in [3], where the concept of gain regions was used to characterize the PO transmit strategies. In [4], the problem of joint maximization of a common utility function with respect to beamforming vectors was studied. It was shown that the problem of finding important PO operating points is NP hard in general. In [5], we proposed an optimization problem that jointly finds the PO pairs of beamforming vectors. There, we maximized the SINR of one link, for a given value of the other, and showed that the optimization problem is quasi-convex. Then, we derived an efficient solution by solving a small number of convex feasibility problems, each having as variables the $n$-dimensional complex beamforming vectors. In [6], it was shown that each point on the Pareto boundary can be found in a decentralized manner. Here, each TX maximizes the rate of its link subject to interference-power constraints.

For the single-input single-output (SISO) IC, the pairing of the PO strategies is well known [7]. The extension to the MISO IC is not straightforward. To find the relation, we build upon the previous approaches to derive a direct condition for PO. Specifically, we start with the optimization problem in [5] and exploit the parameterization in [1]. We propose a new optimization which is the outcome of the Karush-Kuhn-Tucker (KKT) conditions. This optimization is with respect to two real scalars, i.e., the transmit strategy parameters. The novel solution is found in closed form; it only requires solving a cubic equation.

Contributions: In this work we provide a computationally efficient method to compute the Pareto boundary using only one real-valued parameter. ${ }^{1}$ The only computation involved is to solve a cubic equation. The result is validated by numerical illustrations, where we compare the result with the results of the previous works $[1,5]$. This paper is reproducible research and the source code for generating the numerical results is available at urn.kb.se/resolve?urn=urn: nbn: se: liu:diva-64273.

\section{SYSTEM MODEL}

We assume that transmissions consist of scalar coding followed by beamforming and that all propagation channels are frequency-flat. The matched-filtered symbol-sampled complex baseband data received by $\mathrm{RX}_{1}$ is modeled as ${ }^{2}$

$$
y_{1}=\mathbf{h}_{11}^{H} \mathbf{w}_{1} s_{1}+\mathbf{h}_{21}^{H} \mathbf{w}_{2} s_{2}+e_{1},
$$

${ }^{1}$ After submitting this work we became aware of the concurrent work [8] that independently obtained a one-parameter description of the Pareto boundary that is equivalent to the one that we propose here.

${ }^{2}$ The expressions are introduced with respect to link 1 . The expressions for link 2 are obtained by interchanging indices. 
where $\mathbf{h}_{11}, \mathbf{h}_{21} \in \mathbb{C}^{n}$ are the (conjugated) channel vectors of the direct channel $\mathrm{TX}_{1} \rightarrow \mathrm{RX}_{1}$ and cross-talk channel $\mathrm{TX}_{2} \rightarrow \mathrm{RX}_{1}$ respectively. Also, $\mathbf{w}_{1}, \mathbf{w}_{2} \in \mathbb{C}^{n}$ are the beamforming vectors employed by $\mathrm{TX}_{1}$ and $\mathrm{TX}_{2}$, respectively, $s_{1}, s_{2} \sim \mathcal{C N}(0,1)$ are the transmitted symbols of $\mathrm{TX}_{1}$ and $\mathrm{TX}_{2}$, respectively, and $e_{1} \sim$ $\mathcal{C N}\left(0, \sigma_{1}^{2}\right)$ models the receiver noise at $\mathrm{RX}_{1}$. The channels are perfectly known at the transmitters.

The transmit power is bounded due to regulatory and hardware constraints, such as amplifiers. Without loss of generality, we set this bound to 1 and define the set of feasible beamforming vectors as

$$
\mathcal{W} \triangleq\left\{\mathbf{w} \in \mathbb{C}^{n} \mid\|\mathbf{w}\|^{2} \leq 1\right\} .
$$

Note that $\mathcal{W}$ is a convex set.

\section{PRELIMINARIES}

Under the assumptions that the transmitters perfectly know the channel vectors, the receivers treat interference as noise, and Gaussian codes of infinite length are used, the achievable instantaneous rate (in bits/channel use) for link 1 is [1]

$$
R_{1}\left(\mathbf{w}_{1}, \mathbf{w}_{2}\right)=\log _{2}\left(1+\gamma_{1}\left(\mathbf{w}_{1}, \mathbf{w}_{2}\right)\right) .
$$

In (3) we have

$$
\gamma_{1}\left(\mathbf{w}_{1}, \mathbf{w}_{2}\right) \triangleq \frac{p_{1}\left(\mathbf{w}_{1}\right)}{q_{1}\left(\mathbf{w}_{2}\right)},
$$

which is the SINR at $\mathrm{RX}_{1}$ where

$$
p_{1}\left(\mathbf{w}_{1}\right) \triangleq\left|\mathbf{h}_{11}^{H} \mathbf{w}_{1}\right|^{2}
$$

is the useful power received by $\mathrm{RX}_{1}$ from $\mathrm{TX}_{1}$, whereas

$$
q_{1}\left(\mathbf{w}_{2}\right) \triangleq\left|\mathbf{h}_{21}^{H} \mathbf{w}_{2}\right|^{2}+\sigma_{1}^{2}
$$

is the interference-plus-noise power in crosstalk link $\mathrm{TX}_{2} \rightarrow \mathrm{RX}_{1}$.

We note that the rate (3) is a function of the beamforming vectors of both transmitters. Therefore, we define the rate region consisting of all feasible rate pairs $\left(R_{1}\left(\mathbf{w}_{1}, \mathbf{w}_{2}\right), R_{2}\left(\mathbf{w}_{2}, \mathbf{w}_{1}\right)\right)$ as

$$
\mathcal{R} \triangleq \bigcup_{\left(\mathbf{w}_{1}, \mathbf{w}_{2}\right) \in \mathcal{W}^{2}}\left(R_{1}\left(\mathbf{w}_{1}, \mathbf{w}_{2}\right), R_{2}\left(\mathbf{w}_{2}, \mathbf{w}_{1}\right)\right) .
$$

We are interested in the Pareto boundary of the region $\mathcal{R}$, because it uniquely defines it. This boundary consists of all the PO points, at which we cannot improve the rate of one link without decreasing the rate of the other link. Graphically, the Pareto boundary is the northeast boundary of the rate region. The formal definition of Pareto optimality is as follows.

Definition 1. A rate pair $\left(R_{1}^{\star}, R_{2}^{\star}\right) \in \mathcal{R}$ is Pareto optimal if there is no other rate pair $\left(R_{1}, R_{2}\right) \in \mathcal{R}$ with $\left(R_{1}, R_{2}\right) \geq\left(R_{1}^{\star}, R_{2}^{\star}\right)$. (The inequality is component-wise.)

Since we are looking for PO points, we can exploit the previously known parameterization of the Pareto boundary. From [1], we know that PO beamforming vectors must fulfill the necessary conditions that the TXs use all available power and the beamforming vectors are linear combinations of the maximum ratio (MR) and zero-forcing $(\mathrm{ZF})$ strategies. That is

$$
\mathbf{w}_{1}^{\mathrm{PO}}\left(\lambda_{1}\right)=\frac{\lambda_{1} \mathbf{w}_{1}^{\mathrm{MR}}+\left(1-\lambda_{1}\right) \mathbf{w}_{1}^{\mathrm{ZF}}}{\left\|\lambda_{1} \mathbf{w}_{1}^{\mathrm{MR}}+\left(1-\lambda_{1}\right) \mathbf{w}_{1}^{\mathrm{ZF}}\right\|}
$$

for $\lambda_{1} \in[0,1]$. In (8) we have

$$
\mathbf{w}_{1}^{\mathrm{MR}}=\underset{\mathbf{w}_{1} \in \mathcal{W}}{\operatorname{argmax}} p_{1}\left(\mathbf{w}_{1}\right)=\frac{\mathbf{h}_{11}}{\left\|\mathbf{h}_{11}\right\|}
$$

and

$$
\mathbf{w}_{1}^{\mathrm{ZF}}=\underset{\mathbf{w}_{1} \in \mathcal{W}, q_{2}\left(\mathbf{w}_{1}\right)=0}{\operatorname{argmax}} p_{1}\left(\mathbf{w}_{1}\right)=\frac{\Pi_{\mathbf{h}_{12}}^{\perp} \mathbf{h}_{11}}{\left\|\boldsymbol{\Pi}_{\mathbf{h}_{12}}^{\perp} \mathbf{h}_{11}\right\|},
$$

where $\boldsymbol{\Pi}_{\mathbf{h}_{12}}^{\perp} \triangleq \mathbf{I}-\mathbf{h}_{12}\left(\mathbf{h}_{12}^{H} \mathbf{h}_{12}\right)^{-1} \mathbf{h}_{12}^{H}$ is the orthogonal projection onto the orthogonal complement of $\mathbf{h}_{12}$ and $\mathbf{I}$ is the identity matrix. Using beamforming vectors from the parameterization (8), the SINR expression (4) becomes

$$
\gamma_{1}\left(\lambda_{1}, \lambda_{2}\right) \triangleq \frac{p_{1}\left(\mathbf{w}_{1}^{\mathrm{PO}}\left(\lambda_{1}\right)\right)}{q_{1}\left(\mathbf{w}_{2}^{\mathrm{PO}}\left(\lambda_{2}\right)\right)}=\frac{p_{1}\left(\lambda_{1}\right)}{q_{1}\left(\lambda_{2}\right)}
$$

where we write the useful power at $\mathrm{RX}_{1}(5)$ as

$$
p_{1}\left(\lambda_{1}\right)=\left\|\mathbf{h}_{11}\right\|^{2} \frac{\left(\alpha_{1} \lambda_{1}+\left(1-\alpha_{1}\right)\right)^{2}}{2 \alpha_{1} \lambda_{1}^{2}-2 \alpha_{1} \lambda_{1}+1}
$$

and the interference-plus-noise power at $\mathrm{RX}_{1}(6)$ as

$$
q_{1}\left(\lambda_{2}\right)=\frac{\left|\mathbf{h}_{21}^{H} \mathbf{h}_{22}\right|^{2}}{\left\|\mathbf{h}_{22}\right\|^{2}} \frac{\lambda_{2}^{2}}{2 \alpha_{2} \lambda_{2}^{2}-2 \alpha_{2} \lambda_{2}+1}+\sigma_{1}^{2} .
$$

The constants $\alpha_{1}$ and $\alpha_{2}$ are defined in Tab. 1. We note that $p_{1}\left(\lambda_{1}\right)$ and $q_{1}\left(\lambda_{2}\right)$ are ratios of quadratic polynomials in $\lambda_{1}$ and $\lambda_{2}$, respectively. Therefore, (11) is a ratio of polynomials in both $\lambda_{1}$ and $\lambda_{2}$.

In Sec. 4, we make use of the derivatives of $p_{1}\left(\lambda_{1}\right)$ and $q_{2}\left(\lambda_{1}\right)$, which are calculated as

$$
\frac{d p_{1}\left(\lambda_{1}\right)}{d \lambda_{1}}=\left\|\mathbf{h}_{11}\right\|^{2} \frac{2 \alpha_{1}\left(2-\alpha_{1}\right)\left(\alpha_{1} \lambda_{1}+\left(1-\alpha_{1}\right)\right)\left(1-\lambda_{1}\right)}{\left(2 \alpha_{1} \lambda_{1}^{2}-2 \alpha_{1} \lambda_{1}+1\right)^{2}}
$$

and

$$
\frac{d q_{1}\left(\lambda_{2}\right)}{d \lambda_{2}}=\frac{\left|\mathbf{h}_{21}^{H} \mathbf{h}_{22}\right|^{2}}{\left\|\mathbf{h}_{22}\right\|^{2}} \frac{2 \lambda_{2}\left(1-\alpha_{2} \lambda_{2}\right)}{\left(2 \alpha_{2} \lambda_{2}^{2}-2 \alpha_{2} \lambda_{2}+1\right)^{2}} .
$$

\section{CONDITION FOR PARETO OPTIMALITY}

This is the core section of the paper. We derive a sufficient condition for PO points. This condition gives a relation between $\lambda_{1}$ and $\lambda_{2}$ that correspond to $\mathrm{PO}$ operating points.

In [5], we noticed that the Pareto boundary is an one-to-one curve in the two-dimensional space. That is, every PO point $\left(R_{1}^{\star}, R_{2}^{\star}\right)$ is uniquely defined when either of the coordinates is known. Based on this, we proposed a method to derive the Pareto boundary by solving the optimization problem of maximizing one rate for a given value of the other rate. Since the instantaneous rate (3) is monotonously increasing with the SINR, we can equivalently state the optimization problem with respect to the SINR's as

$$
\begin{array}{cl}
\operatorname{maximize}_{\lambda_{1}, \lambda_{2}} & \gamma_{2}\left(\lambda_{2}, \lambda_{1}\right), \\
\text { subject to } & \gamma_{1}\left(\lambda_{1}, \lambda_{2}\right)=\gamma_{1}^{\star} .
\end{array}
$$

The optimization problem (16) takes the SINR of $\mathrm{RX}_{1}$, i.e., $\gamma_{1}^{\star}$, as input and returns as optimal value the SINR of $\mathrm{RX}_{2}$, i.e., $\gamma_{2}^{\star}$, which corresponds to the other coordinate of the PO operating point. The optimal solution of (16) is the pair of transmit strategies $\left(\lambda_{1}^{\star}, \lambda_{2}^{\star}\right)$.

In [5], we used (4) to express the SINR in the optimization (16). We showed that it is a quasi-convex problem of $\left(\mathbf{w}_{1}, \mathbf{w}_{2}\right)$. Here, we 
exploit the fact that the optimization (16) yields PO transmit strategies and instead use the expression (11) for the SINR's. The effect is that we get the same optimal value, reducing the search to the parameter space $\left(\lambda_{1}, \lambda_{2}\right)$. For notational convenience, we do not include the bound-constraints on $\lambda_{1}$ and $\lambda_{2}$ in the optimization problem (16), but we declare a solution feasible only if it adheres to them.

Now, we proceed by deriving the KKT conditions of the optimization problem (16). We do not use the KKT conditions to solve (16) as it stands, i.e., to yield specific PO points. But instead we derive a relation between $\lambda_{1}^{\star}$ and $\lambda_{2}^{\star}$. Note that all solutions to the KKT conditions will yield PO points. This is because the Pareto boundary is an one-to-one curve. Hence, the only optimum of (16) will be the global one. The Lagrange function of (16) is [9]

$$
\mathcal{L}\left(\lambda_{1}, \lambda_{2}, \mu\right)=\gamma_{2}\left(\lambda_{2}, \lambda_{1}\right)-\mu\left(\gamma_{1}^{\star}-\gamma_{1}\left(\lambda_{1}, \lambda_{2}\right)\right)
$$

where $\mu$, is the Lagrange multiplier, which is a real-valued scalar. From (16) we derive the KKT-conditions

$$
\begin{aligned}
& \frac{\partial \mathcal{L}\left(\lambda_{1}, \lambda_{2}, \mu\right)}{\partial \lambda_{1}}=\frac{\partial \gamma_{2}\left(\lambda_{2}, \lambda_{1}\right)}{\partial \lambda_{1}}+\mu \frac{\partial \gamma_{1}\left(\lambda_{1}, \lambda_{2}\right)}{\partial \lambda_{1}}=0, \\
& \frac{\partial \mathcal{L}\left(\lambda_{1}, \lambda_{2}, \mu\right)}{\partial \lambda_{2}}=\frac{\partial \gamma_{2}\left(\lambda_{2}, \lambda_{1}\right)}{\partial \lambda_{2}}+\mu \frac{\partial \gamma_{1}\left(\lambda_{1}, \lambda_{2}\right)}{\partial \lambda_{2}}=0 .
\end{aligned}
$$

By combining (18) and (19) we get the relation

$$
\frac{\frac{\partial \gamma_{2}\left(\lambda_{2}, \lambda_{1}\right)}{\partial \lambda_{1}}}{\frac{\partial \gamma_{1}\left(\lambda_{1}, \lambda_{2}\right)}{\partial \lambda_{1}}}=\frac{\frac{\partial \gamma_{2}\left(\lambda_{2}, \lambda_{1}\right)}{\partial \lambda_{2}}}{\frac{\partial \gamma_{1}\left(\lambda_{1}, \lambda_{2}\right)}{\partial \lambda_{2}}}=-\mu \text {. }
$$

In economics, e.g., [10], a relation similar to (20) is known as the fact that the marginal rate of substitution (MRS) at a PO resource allocation is the same for all consumers (here TX-RX pairs).

By inserting (11) into (20) and elaborating the expression we get the following condition for Pareto-optimality:

$$
\underbrace{\frac{p_{2}\left(\lambda_{2}\right)}{q_{1}\left(\lambda_{2}\right)} \frac{\frac{d q_{1}\left(\lambda_{2}\right)}{d \lambda_{2}}}{\frac{d p_{2}\left(\lambda_{2}\right)}{d \lambda_{2}}}}_{\triangleq g\left(\lambda_{2}\right)}=\underbrace{\frac{q_{2}\left(\lambda_{1}\right)}{p_{1}\left(\lambda_{1}\right)} \frac{\frac{d p_{1}\left(\lambda_{1}\right)}{d \lambda_{1}}}{\frac{d \lambda_{2}\left(\lambda_{1}\right)}{d \lambda_{1}}}}_{\triangleq f\left(\lambda_{1}\right)}
$$

We see that the left-hand-side (LHS) and right-hand-side (RHS) of (21) are functions of only $\lambda_{2}$ and $\lambda_{1}$, respectively. We denote the LHS and RHS of (21) as $g\left(\lambda_{2}\right)$ and $f\left(\lambda_{1}\right)$, respectively. We solve (21) by treating $\lambda_{1}$ as the parameter and $\lambda_{2}$ as the variable. That is, we insert specific values for $\lambda_{1}$, say $\lambda_{1}^{\star}$, in (21) and solve

$$
g\left(\lambda_{2}\right)=f\left(\lambda_{1}^{\star}\right)
$$

By elaborating the expression of $g\left(\lambda_{2}\right)$, we can write it as a ratio of two cubic polynomials. Therefore, we can equivalently rewrite (22) as the cubic equation

$$
c_{3} \lambda_{2}^{3}+c_{2} \lambda_{2}^{2}+c_{1} \lambda_{2}+c_{0}=0 .
$$

The coefficients in (23) are specified in Tab. 1. Cubic equations can be solved in closed form [11]. In Tab. 1, we summarize the method for finding the Pareto boundary in an algorithmic manner.

The roots of (23) are three candidates for $\lambda_{2}^{\star}$. Since $\lambda_{1}^{\star} \in[0,1]$, we have the following three cases.

$\lambda_{1}^{\star}=0$ : Then, the RHS of (22) approaches infinity, since the derivative in the denominator of RHS (21) goes to 0 . In this case, $\lambda_{2}=1$ because the LHS of (21) approaches infinity when

$$
\left.\frac{d p_{2}\left(\lambda_{2}\right)}{d \lambda_{2}}\right|_{\lambda_{2}=1}=0 \text {. }
$$

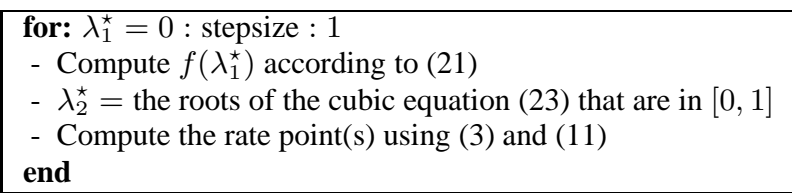

$\alpha_{1} \triangleq 1-\left\|\boldsymbol{\Pi}_{\mathbf{h}_{12}}^{\perp} \mathbf{h}_{11}\right\| /\left\|\mathbf{h}_{11}\right\|$
$\alpha_{2} \triangleq 1-\left\|\boldsymbol{\Pi}_{\mathbf{h}_{21}}^{\perp} \mathbf{h}_{22}\right\| /\left\|\mathbf{h}_{22}\right\|$
$\beta_{2}=\left\|\mathbf{h}_{22}\right\|^{2} \sigma_{1}^{2} /\left|\mathbf{h}_{21}^{H} \mathbf{h}_{22}\right|^{2}$
$c_{0}=\left(\alpha_{2}^{2}-2 \alpha_{2}\right) \beta_{2} f\left(\lambda_{1}^{\star}\right)$
$c_{1}=-\left(2 \alpha_{2}^{3}-3 \alpha_{2}^{2}-2 \alpha_{2}\right) \beta_{2} f\left(\lambda_{1}^{\star}\right)+\left(1-\alpha_{2}\right)$
$c_{2}=\alpha_{2}^{2}+\left(\alpha_{2}^{2}-2 \alpha_{2}\right)\left(1+4 \alpha_{2} \beta_{2}\right) f\left(\lambda_{1}^{\star}\right)$
$c_{3}=-\left(\alpha_{2}^{2}-2 \alpha_{2}\right)\left(1+2 \alpha_{2} \beta_{2}\right) f\left(\lambda_{1}^{\star}\right)-\alpha_{2}^{2}$

Table 1. Description of the method for finding the Pareto boundary.

Also, if $\sigma_{1}^{2}=0$ we can have $\lambda_{2}=0$ since $q_{1}(0)=\sigma_{1}^{2}=0$. This is the scenario of strong interference, for which it is PO if both transmitters use the ZF strategy.

$0<\lambda_{1}^{\star}<1$ : When we get a root of (23) which does not satisfy the feasibility constraint, that $0 \leq \lambda_{2} \leq 1$, we discard it. If we get more than one feasible roots, then all of them correspond to points on the Pareto boundary.

$$
\lambda_{1}^{\star}=1 \text { : Since }
$$

$$
\left.\frac{d p_{1}\left(\lambda_{1}\right)}{d \lambda_{1}}\right|_{\lambda_{1}=1}=0,
$$

the RHS of (22) is 0 . Then, we must have $\lambda_{2}=0$, so that the derivative in the numerator of LHS (21) is 0. Except for the case when $\sigma_{1}^{2} \rightarrow \infty$, where any $\lambda_{2}$ satisfies the condition. This is the noise-limited scenario, for which it is PO if both transmitters use the MR strategy.

\section{NUMERICAL RESULTS}

When consider an exemplary scenario where the transmitters employ $n=3$ antennas each and the channels are

$$
\begin{aligned}
& \mathbf{h}_{11}=[0.698-0.340 i, 0.510-1.36 i,-1.39+1.30 i]^{T}, \\
& \mathbf{h}_{12}=[-0.366+0.700 i, 0.386+0.511 i, 0.207-0.692 i]^{T}, \\
& \mathbf{h}_{21}=[-0.761-1.79 i,-0.825+0.487 i,-1.13-1.64 i]^{T}, \\
& \mathbf{h}_{22}=[0.230+0.336 i, 0.0312+0.297 i, 0.0570-0.203 i]^{T} .
\end{aligned}
$$

We illustrate our result by plotting the rate region and $\lambda_{2}^{\star}$ as a function of $\lambda_{1}^{\star}$. In Fig. 1 we have $\sigma_{1}^{2}=\sigma_{2}^{2}=1$ (low signal-to-noise-ratio (SNR)) and in Fig. 2 we have $\sigma_{1}^{2}=\sigma_{2}^{2}=0.01$ (high SNR).

We sampled $\lambda_{1}$ in 100 points and created the plots using the algorithm in Tab. 1. For validation purposes, we also illustrate the corresponding result using [1]. We sampled the $\left(\lambda_{1}, \lambda_{2}\right)$ space in $100 \times$ 100 points, which gave approximately 100 points on the boundary. In addition, we generated 10 points on the boundary using [5]. We observe that we obtain the same region with the three methods. The merit of the proposed method herein is that we have significant complexity reduction. We avoid the brute-force coupling of [1] and the need to solve convex problems of [5]. In Fig. 1, we see that each $\lambda_{1}^{\star}$ gives a unique $\lambda_{2}^{\star}$, whereas in Fig. 2, we get up to three feasible values for $\lambda_{2}^{\star}$ for some $\lambda_{1}^{\star}$. Note that the right part of Fig. 1 is plotted in linear scale, whereas in Fig. 2 it is plotted in logarithmic scale. 

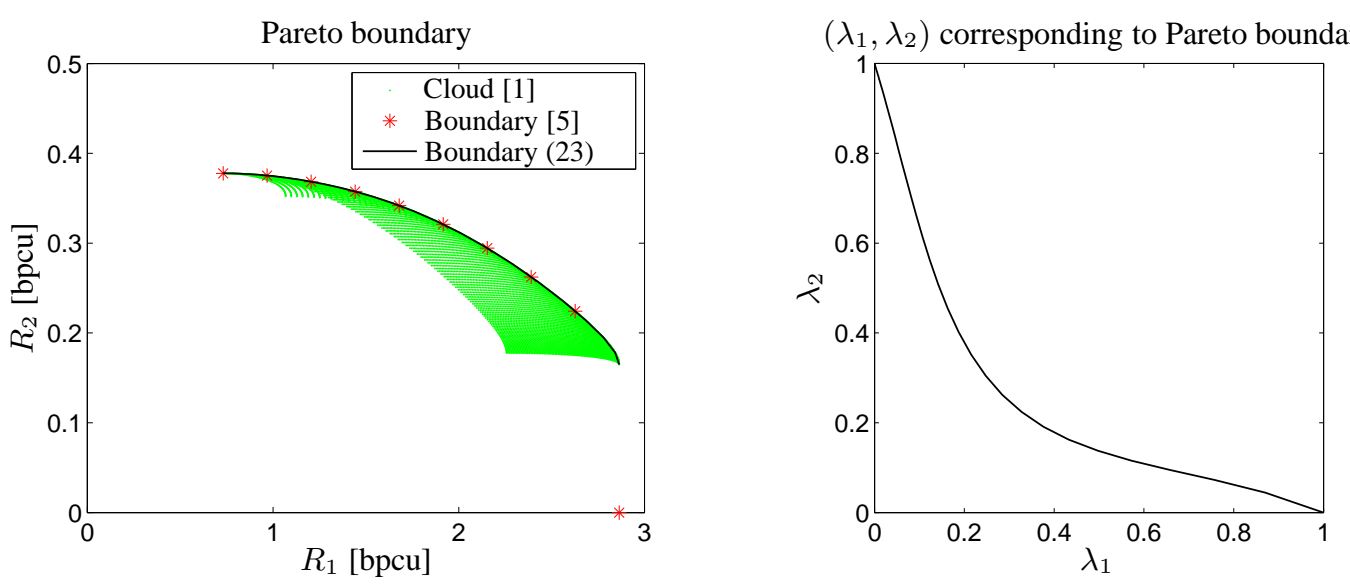

Fig. 1. Rate region and $\mathrm{PO}$ pairs of transmit strategies, $\sigma_{1}^{2}=\sigma_{2}^{2}=1$. The plot to the right is in linear scale.
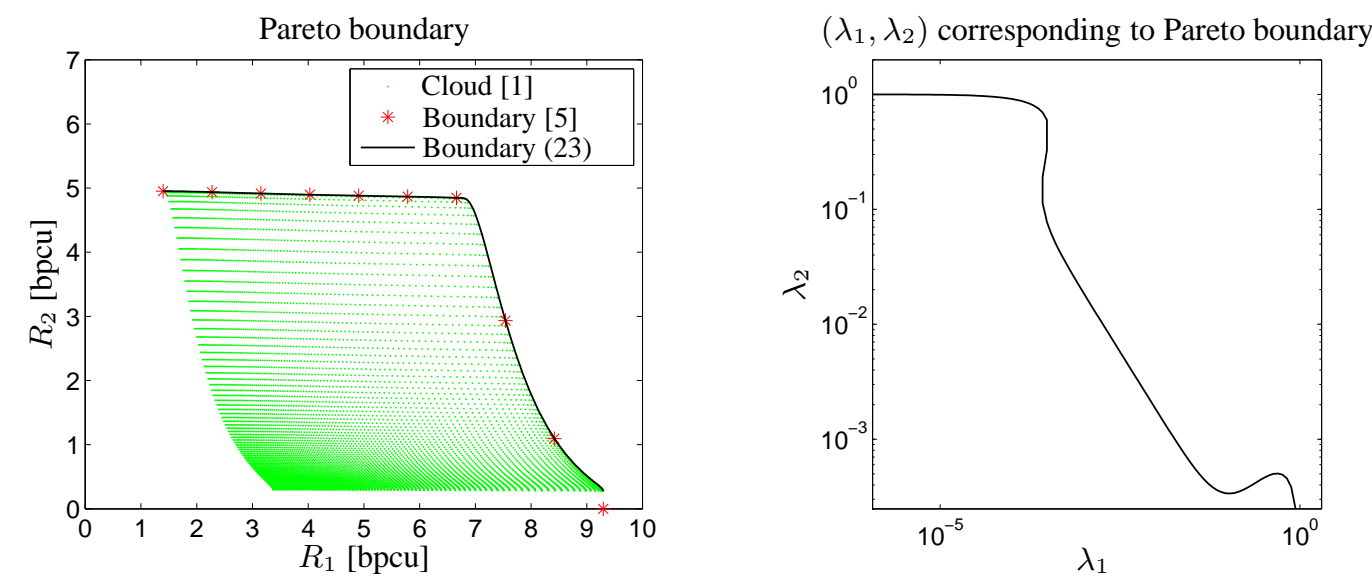

Fig. 2. Rate region and PO pairs of transmit strategies, $\sigma_{1}^{2}=\sigma_{2}^{2}=0.01$. The plot to the right is in logarithmic scale.

\section{CONCLUSIONS}

We proposed a method to efficiently find the Pareto boundary of an achievable rate region for the MISO IC. This method greatly reduces the computational complexity when compared with the state-of-theart approaches. Compared to the characterization in [1], we have effectively limited the search space from two dimensions to one. Instead of using brute-force coupling we solve a cubic equation. The method returns the entire Pareto boundary and cannot be directly used to calculate specific PO points as the one in [5]. We hope that the core result will motivate resource allocation algorithms.

\section{REFERENCES}

[1] E. A. Jorswieck, E. G. Larsson, and D. Danev, "Complete characterization of the Pareto boundary for the MISO interference channel," IEEE Trans. Signal Process., vol. 56, pp. 5292-5296, Oct. 2008.

[2] R. Zakhour and D. Gesbert, "Coordination on the MISO interference channel using the virtual SINR framework," in Proc. ITG Workshop on Smart Antennas (WSA), 2009.

[3] R. Mochaourab and E. A. Jorswieck, "Optimal beamforming in interference networks with perfect local channel information," IEEE Trans. Signal Process., vol. 59, pp. 1128-1141, Mar. 2011.
[4] Y.-F. Liu, Y.-H. Dai, and Z.-Q. Luo, "On the complexity of optimal coordinated downlink beamforming," in Proc. IEEE ICASSP, Dallas, TX, Mar. 2010, pp. 3274 -3277.

[5] E. Karipidis and E. G. Larsson, "Efficient computation of the Pareto boundary of the MISO interference channel with perfect CSI," in Proc.WiOpt, 2010, pp. 573-577.

[6] R. Zhang and S. Cui, "Cooperative interference management in multi-cell downlink beamforming," IEEE Trans. Signal Process., vol. 58, pp. 5450-5458, Oct. 2010.

[7] M. Charafeddine, A. Sezgin, and A. Paulraj, "Rate region frontiers for n-user interference channel with interference as noise," in Proc. Allerton, 2007.

[8] R. Mochaourab and E. Jorswieck, "Walrasian equilibrium in two-user multiple-input single-output interference channels," in Proc. IEEE ICC, 2011.

[9] S. Boyd and L. Vandenberghe, Convex Optimization, Cambridge University Press, 2004.

[10] D. G. Luenberger, Microeconomic Theory, McGraw-Hill, 1995.

[11] M. Abramowitz and I. A. Stegun, Handbook of Mathematical Functions with Formulas, Graphs, and Mathematical Tables, National Bureau of Standards, 1972. 